\title{
Elaboración de estándares de la fuerza de agarre en individuos sanos entre 20 y 70 años residentes en la localidad de Usaquén, Bogotá
}

Olga Lucía Hincapié G.

\section{Resumen}

El propósito de la presente investigación fue elaborar los estándares de la fuerza de agarre en individuos sanos entre 20 y 70 años residentes de la localidad de Usaquén en Bogotá. Se evaluó la fuerza de agarre en una muestra aleatoria estratificada de ambos géneros conformada por 385 personas de los 6 estratos socioeconómicos. Este trabajo es de tipo descriptivo evaluativo. Se utilizaron 2 posiciones de medida, la primera denominada A es la que describe la Sociedad Americana de Cirugía de la Mano con el codo en $90^{\circ}$ de flexión; la se gunda denominada posición B en la que el codo está extendido en $0^{\circ}$. Para realizar las mediciones se utilizó el dinamómetro Jamar. Como resultados del trabajo se presentan dos tablas con los estándares de la fuerza del agarre en las dos posiciones para la muestra de población colombiana y una tabla comparativa con los valores de la población norteamericana.

Palabras clave: fuerza, agarre, evaluación, medición.

\section{Elaboration of grip strenght standards in healthy persons, 20 to 70 years of age, living in Usaquen}

\section{Abstract}

The purpose of the present investigation is to establish standards of the grip strength in healthy individuals between 20 and 70 years old residents in Usaquén in Bogotá. The grip strength was evaluated in a stratified random samples of both gender conformed by 385 people of the 6 socioeconomic strata. This work is of descriptive and evaluative type. Two measured positions were used, the first one denominated $A$ is the one that describes the American Society of Surgery of the Hand, the second denominated position $B$ in which the elbow is extended in $0^{\circ}$. The instrument used to carry out the measurements of grip strength was the Jamar dynamometer. Two charts were created as a result of the investigation, one for the Colombian population's values and a comparative one with the North American population's values.

Key words: grip strength, evaluation, measurement.

* Fisioterapeuta, Especialista en Rehabilitación de Mano y Miembro Superior. olgaluciahin@ @otmail.com 


\section{Introducción}

La medida de la fuerza del agarre ha sido uno de los puntos clave dentro de la evaluación general de la mano. Se han elaborado estándares con respecto a esta medida, y de hecho, se cuenta con tablas descritas en poblaciones según género, edad, preferencia manual y posición del codo; sin embargo, dichas tablas pertenecen a población extranjera de modo que en nuestro país no se cuenta aún con estándares propios.

Teniendo en cuenta que elaborar estándares de la fuerza de agarre de toda la población colombiana representaría un proyecto muy ambicioso con elevados costos, se desarrolló una primera etapa en la que se recopilaron datos y elaboraron los estándares de la fuerza de agarre en una muestra de población sana de la localidad de Usaquén residentes en Bogotá, con un rango de edad de 20 a 70 años, considerando que esta localidad corresponde al área de influencia más inmediata geográficamente de la Institución Universitaria Fundación Escuela Colombiana de Rehabilitación.

La fuerza se define como la tensión que un músculo puede oponer a una resistencia en un solo esfuerzo máximo. El tener mediciones de la fuerza de agarre en la muestra mencionada constituye una base sobre la cual se pueden apoyar futuros estudios relacionados con requerimientos de la fuerza, para desempeño de los diferentes roles del ser humano en su vida diaria.

Entre los estudios recientes se encuentra el de Chwen (1994), en el que se investigó el efecto de la posición del hombro en la fuerza de agarre en 80 hombres y 80 mujeres. Para mediar la fuerza de agarre en cuatro posiciones se utilizó un dinamómetro marca Jamar. Las cuatro pruebas consistían en tres posiciones en las cuales el codo era mantenido en extensión completa combinada con grados variables de flexión de codo entre $0^{\circ}, 90^{\circ}$ y $180^{\circ}$, y en una posición en la que el codo era flejado c $90^{\circ}$ con el húmero en $0^{\circ}$ de flexión. Solamente se evaluó la mano dominante y se registró la medida de fuerza de agarre más alta con el hombro colocado a $180^{\circ}$ de flexión con el codo en extensión completa, mientras que la fuerza de agarre más baja se presentó en la posición de $90^{\circ}$ de flexión de codo con el hombro en $0^{\circ}$ de flexión.

La fuerza de agarre medida con el codo en extensión, independientemente de la posición del hombro, fue más significativa que cuando el codo era flexionado a $90^{\circ}$ con el hombro en posición de $0^{\circ}$ de flexión. Su análisis varía con respecto al género y para cada grupo de edad; siendo para los hombres mayor entre los 20 y 30 años, y para las mujeres entre 40 y 49 años, decreciendo proporcionalmente con la edad.

De acuerdo con el estudio realizado por Kuzala y Vargo (1992), existe relación entre la posición del codo y la fuerza de agarre, medida con el codo en $0,45,90$ y $135^{\circ}$ de flexión, en una muestra de estudiantes, siendo el nivel de fuerza significativamente mayor en $0^{\circ}$ y menor en $135^{\circ}$ de flexión.

En un estudio realizado por Remanen en 1999 se encontró que la fuerza de agarre de la mano disminuye con la edad y predispone a la persona sana a limitaciones funcionales.

Balogun (1991) diseñó un estudio para determinar los efectos de la postura y de la posición del codo en la fuerza del agarre en 26 mujeres y 35 hombres en cuatro posiciones:

1. Sentado con el codo en $90^{\circ}$ de flexión.

2. Sentado con el codo en completa extensión.

3. De pie con el codo en $90^{\circ}$ de flexión.

4. De pie con el codo en completa extensión.

El análisis reveló que los hombres tienen mayor fuerza de agarre que las mujeres en todas las posiciones y en todos los ángulos de posición del codo, y hubo una diferencia estadísticamente significativa entre la posición sentada con el codo en $90^{\circ}$ de flexión y de pie con el codo en completa extensión. 
Desrosiers y Bravo (1995) plantean que el agarre disminuye curvilinealmente con la edad y el hombre es considerado más fuerte que la mujer.

Kellor, Frost y Silverberg (1971), describieron el desarrollo de datos normales para la fuerza de agarre en personas mayores de 60 años. Se evaluaron 360 personas con el dinamómetro de Jamar y el vigorímetro de Martín según el Protocolo de la Sociedad Americana de Terapeutas de Mano, donde se concluyó que la fuerza de agarre disminuye con la edad y los hombres son consistentemente más fuertes que las mujeres. Además de la edad y del género, la altura y el diámetro de la mano, demostraron ser los mejores indicadores de la fuerza de agarre para esta población de adultos mayores. La fuerza de agarre ha sido correlacionada con otras variables tales como la estatura, el peso, la dominancia y el estado físico general. Se ha encontrado que ésta se asocia más con el peso que con la estatura, y que, por consiguiente debe haber una asociación más cercana con la masa muscular que con el tamaño de esqueleto en general.

En Colombia, Giraldo (2003) realizó un estudio sobre fuerza de agarre en 150 hombres ancianos en Manizales, edad promedio fue de 70.5 años, encontrando que el promedio de la fuerza de agarre fue de $31 \mathrm{~kg} /$ fuerza, con un rango de rango de 15 a $48 \mathrm{~kg} /$ fuerza. Los resultados de este estudio permiten concluir que la pérdida de fuerza está acentuada en $1.4 \%$ entre los 60 y 85 años, acentuándose aún más después de los 80 .

La ocupación también influye en la fuerza de agarre. Josty en 1997, realizó un estudio de la fuerza de agarre en 104 sujetos cuyas ocupaciones están relacionadas con el trabajo manual ligero, pesado y trabajo no manual. Los resultados de este estudio revelaron que las personas con trabajo manual pesado tuvieron mayor fuerza que los de trabajo manual de oficina y los de trabajo manual liviano estuvieron en medio de estos dos grupos de estudio. Consecuentemente, el autor recomienda que la ocupación del paciente debe ser tenida en cuenta para las mediciones de la fuerza de agarre necesarios en la rehabilitación y en el reporte médico legal.

La dominancia de la extremidad es también un factor importante en el estudio de la fuerza de agarre. Petersen (1989) realizó estudios respecto a la fuerza del agarre en la mano dominante y la no dominante en personas diestras y zurdas. Menciona que la mano dominante posee $10 \%$ más de fuerza de agarre que la mano no dominante. Este estudio, realizado con 310 hombres y mujeres, muestra una diferencia de $10,74 \%$ si la mano dominante es la derecha. Para las personas con dominancia en la mano izquierda la fuerza de agarre es equivalente en ambas manos.

Sin embargo, un estudio realizado con población de 30 a 50 años, donde la mujer tenía el 60 a $70 \%$ menos de fuerza que los hombres, mostró resultados no significativos en relación con la dominancia manual (Harkonen, 1993).

Nurgul (2002), describe también el efecto de la dominancia de la mano en la fuerza de agarre. El estudio fue diseñado para evaluar la fuerza de agarre y de la pinza en una población de dominancia diestra y zurda. Se incluyeron 128 personas diestras y 21 zurdas. Los resultados establecieron que la fuerza de agarre es mayor en la mano dominante de los sujetos diestros y que hay una diferencia estadísticamente significativa entre la fuerza de agarre y pinza de la mano dominante y la no dominante, a favor de la dominante. Se concluyó que la mano dominante es significativamente más fuerte en sujetos diestros pero no hubo diferencia significativa en la población zurda. Para la realización de este trabajo de investigación se revisaron una serie de estudios efectuados en diferentes países con el fin de intentar establecer parámetros de fuerza de agarre.

Entre éstos están los estudios de Mathiowetz quien en 1985 en Milwaukee realizó un estudio para establecer datos normales de la fuerza de aga- 
rre y pinza en adultos entre los 20 y 75 años. Se analizaron 310 hombres y 328 mujeres mostrando los puntajes más altos en la fuerza de agarre entre el grupo de 25 a 39 años, lo que confirma la gran correlación entre la fuerza de agarre y la edad. Se ha encontrado que la fuerza de agarre promedio en otros países es de 20 libras y que éste es el promedio mínimo para poder realizar las actividades básicas cotidianas, se sustenta que las personas tienen un $10 \%$ más de fuerza en la mano dominante que en la mano no dominante (Hunter, 1995).

Para abordar el problema de la fuerza de agarre es importante mencionar la clasificación de la prensión que hace Kapandji (1998). Este autor clasifica la prensión en tres grupos: las presas digitales, las presas palmares, las presas centradas y las presas digitales, las cuales se dividen a su vez en dos grupos: las presas y las presas pluridigitales.

Las presas digitales constituyen la clásica pinza pulgardigital, generalmente pulgar índice. Asimismo, son de tres tipos dependiendo de que la oposición sea terminal, subterminal o subterminolateral.

La presa por oposición terminal o término pulpejo es la más fina y precisa. Permite sujetar un objeto de pequeño calibre, coger muy fino. El pulgar y el índice (o el medio) se oponen en el extremo del pulpejo e inclusive en los casos de algunos objetos extremadamente finos por el borde de la uña. Por lo tanto, se necesita un pulpejo elástico y correctamente apuntalado por la uña, cuya función primordial es este tipo de prensión. Por este motivo, también se puede denominar prensión pulpejo ungueal. Es la prensión más fácil de comprometer ante la menor alteración de la mano; de hecho, precisa de un juego máximo articular y sobre todo de la integridad de los puntos musculares y tendones, y en particular: el flexor profundo y el flexor largo propio del pulgar (figura 1).

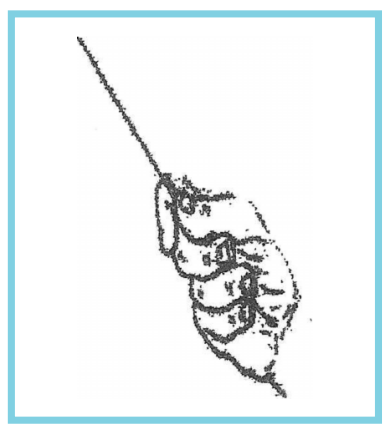

Figura 1. Prensión pulpejo ungueal.

La prensión por oposición subterminal o del pulpejo es el tipo más común. Permite sujetar objetos relativamente más gruesos. El test de la eficacia de la prensión de pulpejo subterminal en intentar arrancar una hoja de papel sujeto con firmeza por el pulgar y el índice. Si la oposición es buena, la hoja no se puede arrancar (signo de Froment). Los principales músculos de este tipo de presión son: el flexor superficial, los músculos tenares flexores de la primera falange del pulgar (flexor corto, primer interóseo palmar, abductor corto y sobre todo el aductor).

La presa por oposición subterminotaterai o pulpejo lateral, es un tipo de prensión que puede suplir la oposición terminal o la subterminal cuando se han amputado las dos últimas falanges del índice: la presa es menos fina aunque sigue siendo sólida. La cara palmar del pulpejo del pulgar contacta con la cara externa de la primera falange del índice. Los músculos más importantes de este tipo de prensión son: el primer interóseo dorsal, el flexor corto, el primer interóseo palmar y sobre todo el aductor del pulgar (figura 2).

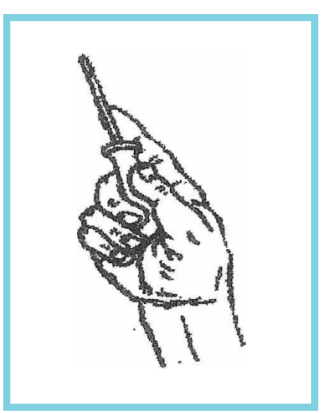

Figura 2. Presa por oposición subtérmino lateral o pulpejo lateral. 
Entre las presas digitales, existe una que no constituye una pinza pulgar-digital, esta es la prensión interdigital lateral, es un tipo de prensión accesoria. Generalmente, se efectúa entre el índice y el medio, el pulgar no interviene. El diámetro del objeto que se ha de coger debe ser pequeño. Los músculos que participan son los interóseos (segundos interóseos palmar y dorsal), es una presa débil y sin precisión.

Las presas pluridigitales hacen intervenir además del pulgar, los otros dos, tres o cuatro dedos; permiten una prensión mucho más firme que la bidigital que persiste como presa de precisión; las presas tridigitales comprometen al pulgar, índice y medio y son las que se utilizan con mayor frecuencia. Se utiliza esta presa para llevarse los alimentos a la boca. Se asemeja entonces a la presa tridigital del pulpejo. Por ejemplo, escribir con un lápiz, necesita una presa interdigital, del pulpejo en el caso del índice y del pulgar, y lateral para la tercera falange del medio que el fondo de la primera comisura (figura 3 ).

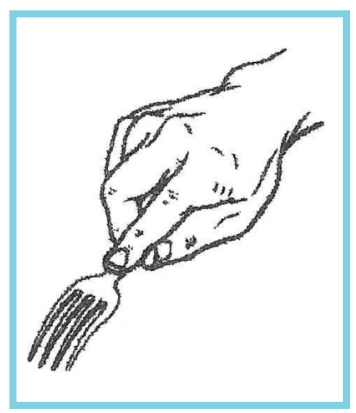

Figura 3. Presa tridigital.

Las presas tetradigitales se utilizan cuando se trata de un objeto muy grueso que se debe coger con mayor firmeza. La firmeza puede ser tetradigital del pulpejo, cuando coge un objeto esférico, en este caso se puede observar que el contacto se lleva a cabo por el pulpejo en el caso del pulgar, índice y medio, mientras que el lateral en el caso de la tercera falange del anular, cuya función es evitar que el objeto se escape hacia adentro. Tetradigital pulpejo-lateral, cuando se desenrosca una tapa.
En este caso, el contacto del pulgar es amplio abarcando el pulpejo y la cara palmar de la primera falange, así como sobre el índice y el medio; es lateral y del pulpejo en la segunda falange del anular que bloquea el objeto por dentro. Tetradigital del pulpejo pulgardigital, como cuando se sostiene un lápiz, donde el pulpejo del pulgar dirige y mantiene el objeto cuando con fuerza contra el pulpejo del índice, del medio y del anular casi en máxima extensión (figura 4).

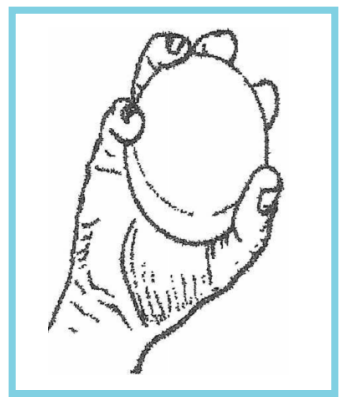

Figura 4. Presa pentadigitales.

Las presas pentadigitales emplean todos los dedos, el pulgar se opone de forma variada a los otros dedos. Se utiliza generalmente para coger grandes objetos. Sin embargo, cuando se trata de un objeto pequeño, se puede coger con una presa pentadigital del pulpejo, de modo que sólo el quinto dedo efectúa un contacto lateral. Si el objeto es un poco más voluminoso, la presa se convierte en pentadigital pulpejo-lateral; los cuatro primeros dedos conectan con toda su cara palmar y envuelven el objeto casi totalmente, el pulgar se opone a los tres otros dedos y el meñique evita mediante su cara externa cualquier posible desplazamiento del objeto hacia dentro y en sentido proximal.

Otra presa pentadigital que se podría denominar pentadigital comisural, coge objetos gruesos semiesféricos, como por ejemplo un cuenco, envolviéndolo con la primera comisura: pulgar e índice ampliamente extendidos y separados conectan con toda su cara palmar, lo que precisa una gran flexibilidad y posibilidades normales de separación de la primera comisura. La presa pentadigital "panorámica" permite coger grandes 
objetos planos, una bandera por ejemplo. Para poder realizarla se necesita una gran separación de los dedos ampliamente divergente, el pulgar se coloca en retroposición y en máxima extensión, de modo que esté en máxima contraposición. La eficacia de esta presa depende de la integridad de las interfalángicas distales y de la acción de los flexores profundos.

Las presas palmares hacen intervenir, además de los dedos, la palma de la mano. Son de dos tipos según se utilice o no el pulgar (figura 5).

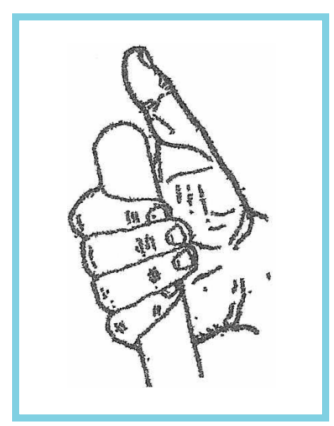

Figura 5. Presa palmar.

La prensión dígito palmar opone la palma de la mano con los últimos cuatro dedos. Es un tipo de presa accesoria pero utilizada con frecuencia cuando se maneja una palanca o sujeta un volante que permite que el pulgar recorra una directriz, es decir un círculo ? el camino más corto para dar la vuelta. Por otra parte, el volumen del objeto exige la máxima libertad de separación de la primera comisura.

Las presas palmares esféricas pueden implicar tres, cuatro o cinco dedos. Cuando interviene tres o cuatro dedos el último dedo implica por dentro, bien sea el medio en la presa esférica tridigital, o el anular en la presa esférica tetradigital, contactan por la cara lateral externa con el objeto, constituye así un tope interno, reforzado por dos dedos restantes. Este tope se opone a la presión del pulgar de modo que el objeto queda bloqueado distalmente por los ganchos de los dedos que establecen un contacto palmar con el objeto. En la presa palmar esférica pentadigital todos los dedos contactan con el obje- to por su cara palmar. El pulgar se opone al anular; en conjunto ocupan el mayor diámetro y el bloqueo de la presa está asegurada distalmente por el índice y el medio y, proximalmente por la eminencia tenar y el meñique. El objeto se sujeta con firmeza por todos los dedos en forma de gancho. Esta presa es mucho más simétrica que las dos anteriores y, en este sentido, constituye la transición con las siguientes.

Las presas centradas realizan, de hecho, una simetría en torno al eje longitudinal que, en general, se confunde con el eje del antebrazo. En cualquier caso el objeto de forma alargada se coge con firmeza mediante una presa palmar donde intervienen el pulgar y los últimos tres dedos. El índice, en este caso, desempeña una función orientadora indispensable para dirigir el utensilio.

Las presas centradas o direccionales se utilizan con frecuencia; requieren de la integridad de la flexión de los últimos tres dedos, la extensión completa del índice cuyos flexores deben ser eficaces, y un mínimo de oposición del pulgar para el cual la flexión de la interfalángica no es indispensable (figura 6).

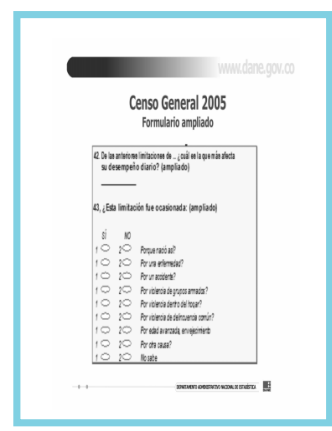

Figura 6. Presas centradas.

El método más utilizado para medir la fuerza es el test muscular manual. Este test es limitado debido a la subjetividad de la técnica por los errores en el juicio humano. Es por esto, que como parte de la evaluación de la musculatura intrínseca y extrínseca de la mano se hace la determinación de la fuerza del agarre en forma comparativa, utilizando el dinamómetro Jamar, desarrollado por 
Bechtol y recomendado por la Sociedad Americana de Cirugía de Mano (Hunter, 1995).

El método por el cual se mide la fuerza de prensión es la dinamometría manual, dando la capacidad de los individuos para coger con firmeza los objetos. Según la Sociedad Americana de Terapia de la Mano, consiste en un instrumento hidráulico con un mango sensible calibrado en libras y kilogramos y es considerado como el instrumento más preciso y confiable para la medición objetiva de la fuerza del agarre. Este instrumento posee cinco posiciones para ajustar el mango del agarre 1, 1.5, 2, 2.5 y 3 pulgadas (figura 8).

El paciente es instruido con respecto a la posición en que debe colocar su miembro superior: el antebrazo debe estar en neutro, el codo flexionado a $90^{\circ}$, el brazo adosado al tronco. La muñeca debe estar entre 0 y $30^{\circ}$ de extensión y 0 a $15^{\circ}$ de desviación cubital. La fuerza es medida en cada una de las cinco posiciones del dinamómetro alternando la mano izquierda y derecha respectivamente. Se ha dicho que existe de un 5 a un $10 \%$ de diferencia entre la mano no dominante y la mano dominante.

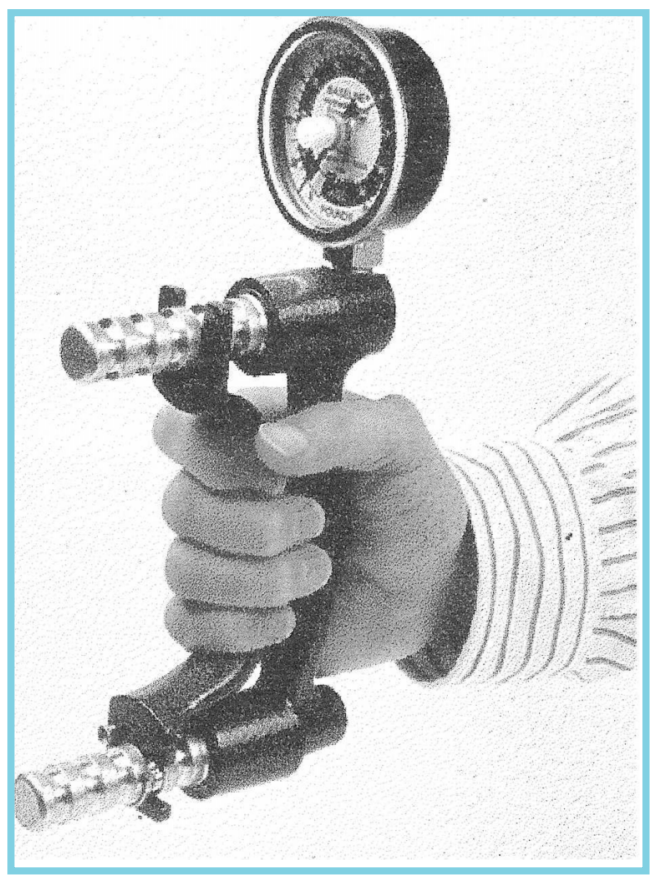

Figura 8. Dinamómetro.
Como recomendación de la (ASSH), (American Society for Surgery of the Hand), para evitar la influencia de la fatiga, se somete a cada paciente a la realización de la prueba en cada posición del dinamómetro y repitiendo la prueba tres veces en cada posición. Se ha aceptado realizar la prueba en la segunda posición del dinamómetro alternando las dos manos, tres veces en cada una y sacando el promedio de las tres mediciones. (Kuzala y Vargo, 1992).

El objetivo general de este trabajo fue elaborar los estándares de la fuerza de agarre en individuos sanos de ambos sexos entre 20 y 70 años, nacidos en Colombia, residentes en la localidad de Usaquén en Bogotá.

Los objetivos específicos señalados en esta investigación fueron:

- Establecer los estándares de la fuerza de agarre según género en personas de 20 a 70 años.

- Establecer los estándares de la fuerza de agarre de la mano dominante en personas de 20 a 70 años.

- Establecer los estándares de la fuerza de agarre de la mano no dominante en personas de 20 a 70 años.

- Establecer los estándares de la fuerza de agarre en la posición de codo en flexión de $90^{\circ}$ (posición A) en personas de 20 a 70 años.

- Establecer los estándares de la fuerza de agarre en la posición de codo en extensión de $0^{\circ}$ (posición B) en personas de 20 a 70 años.

- Describir el promedio de la fuerza de agarre en relación con el peso y el género.

- Describir el promedio de la fuerza de agarre en relación con la talla y el género.

- Describir el promedio de la fuerza de agarre en relación con la ocupación y el género. 


\section{Variables descriptoras:}

Fuerza de agarre con codo flexionado: con base en la Asociación Americana de Cirugía de la Mano se utiliza la posición A con codo en $90^{\circ}$ de flexión.

Fuerza de agarre con codo extendido: posición $\mathrm{B}$ con el codo en $0^{\circ}$ de extensión.

\section{Variables intervinientes:}

Género: diferencia física y constitutiva del hombre y de la mujer. Se obtiene mediante la observación directa.

Edad: tiempo transcurrido desde el nacimiento. Ésta se obtiene mediante las preguntas realizadas en la encuesta.

Preferencia manual: utilización de un miembro superior con mayor frecuencia. Se obtiene mediante las preguntas realizadas en la encuesta.

Ocupación: actividad o labor la cual realiza una persona.

Talla: Medida tomada como punto de comparación en unidades determinadas. Ésta se halla mediante la utilización de una cinta métrica de $200 \mathrm{~cm}$, ubicando la persona en posición anatómica, apoyado en la pared.

Peso: Es la fuerza ejercida por un individuo sobre la tierra debido a la aceleración de la gravedad expresada en kilogramos. Éste se obtiene mediante una báscula.

\section{Metodología}

\section{Tipo de investigación:}

Esta investigación es de tipo descriptivo.

\section{Participantes:}

Para el desarrollo de la investigación se tomó una muestra representativa de 385 personas de ambos géneros, el $51.69 \%$ femenino y el $48.31 \%$ masculino, que no presentaran ninguna alteración de miembros superiores, entre 20 y 70 años de edad nacidos en Colombia, residentes en la localidad de Usaquén en Bogotá y de todos los estratos socioeconómicos.

No se incluyeron en el estudio personas que tuvieran algún tipo de alteración de miembros superiores, personas menores de 20 y mayores de 70 años, personas residentes en una localidad diferente a la de Usaquén, personas que practicasen deportes de alto rendimiento y personas que estuvieran bajo los efectos de medicamentos depresores del sistema nervioso o relajantes musculares.

La selección de la muestra se llevó a cabo de acuerdo con la estimación de población de la localidad por estrato socioeconómico realizado por el Departamento Administrativo de Planeación Distrital para el año 2003 obteniéndose la totalidad de la población residente en la localidad de Usaquén. Se hizo un muestreo no probabilístico estratificado.

Los datos específicos de la muestra se presentan en la tabla 1.

\section{Tabla 1}

ESPECIFICACIONES DE LA MUESTRA POR ESTRATOS SOCIOECONÓMICOS

\begin{tabular}{c|c}
\hline Estrato & Tamaño de la muestra \\
\hline 1 & 14 \\
2 & 38 \\
3 & 110 \\
4 & 98 \\
5 & 55 \\
6 & 70 \\
Total & 385 \\
\hline
\end{tabular}

\section{Instrumentos:}

Para la evaluación inicial fue necesaria la anamnesis incluida dentro del formato de evaluación: 
- Dinamómetro Jamar (de la Escuela Colombiana de Rehabilitación).

- Goniómetro.

- Báscula.

- Cinta métrica.

\section{Procedimiento:}

Se obtuvo la información mediante una evaluación de 11 preguntas. Se realizó de manera aleatoria en la población sana de la localidad de Usaquén en Bogotá; a cada participante se le informó de manera global el propósito y la metodología del estudio.

Con el dinamómetro se midió la fuerza de agarre con el codo en $90^{\circ}$ de flexión que se denominó posición $\mathrm{A}$ y con el codo extendido en $0^{\circ}$ que se denominó posición $\mathrm{B}$.

Para la recolección de la muestra se contó con la colaboración de todos los estudiantes que rotaron por la práctica de mano del Hospital Militar Central durante el año 2004 quienes se hicieron cargo de recolectar la muestra requerida. Las personas que cumplieron con los requisitos de inclusión procedieron a diligenciar el consentimiento informado y posteriormente fueron evaluados utilizando un dinamómetro Jamar en posición 2 de acuerdo con lo establecido por la Sociedad Americana de Cirugía de la Mano. A cada participante se le dieron las siguientes instrucciones: colocarse en posición sedente, con la cabeza en neutro y mirando al frente. Se tomaron las medidas con el codo en dos posiciones diferentes. Posición A: Brazo en aducción, $90^{\circ}$ de flexión de codo, antebrazo en neutro sin apoyo. Posición B: $90^{\circ}$ de flexión de hombro, $0^{\circ}$ de extensión de codo, antebrazo en neutro sin apoyo. Cada participante fue previamente pesado en una báscula y medido con la cinta métrica para establecer peso y talla.

Se colocó el dinamómetro primero en la mano dominante y se le pidió que ejerciera presión lo más fuerte posible; luego se hizo en la mano no dominante. Se repitió el procedimiento en forma alterna tres veces para cada mano y se anotaron los valores de cada medición y el promedio de las tres mediciones.

\section{Resultados}

Inicialmente se presentan dos gráficas con la fuerza de agarre comparativa entre hombres y mujeres, tanto con la mano derecha como con la mano izquierda, observándose que la fuerza de agarre es mayor en hombres que en mujeres y que en el género masculino se presenta un pico de mayor fuerza de agarre entre los 30 y 34 años y en las mujeres de los 35 a los 39 años. Se comprueba también que la fuerza del agarre disminuye progresivamente con el aumento de la edad (gráficas 1 y 2).

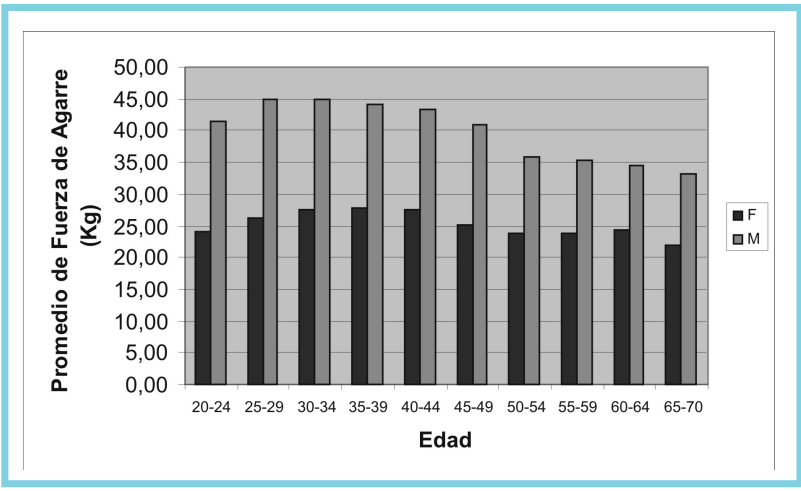

Gráfica1. Promedios fuerza de agarre Posición A - Mano derecha

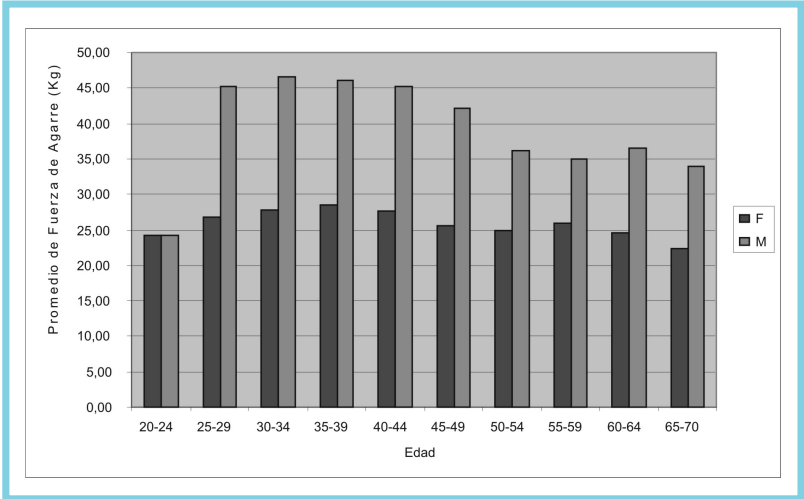

Gráfica 2. Promedios fuerza de agarre Posición B - Mano derecha 
Con relación a la dominancia en el género femenino se observa mayor población diestra que zurda y un comportamiento en cuanto a la fuerza de agarre más consistente en las diestras (gráfica 3).

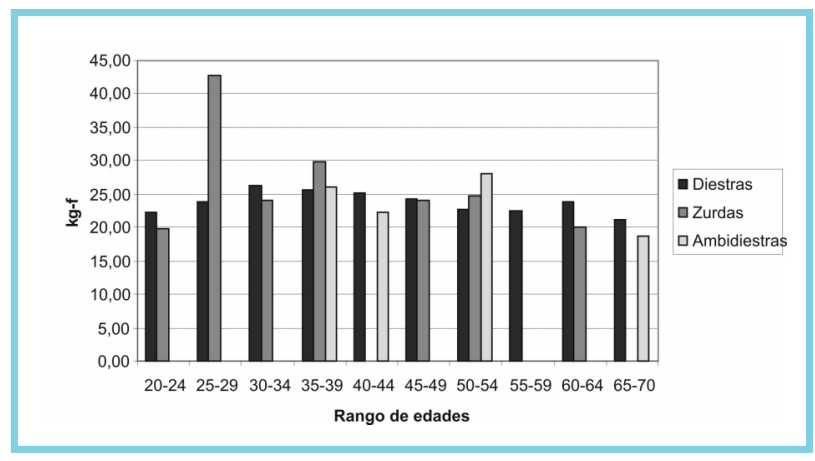

Gráfica 3. Fuerza en posición A de la mano izquierda en mujeres por dominancia

En el género masculino hay mayor población de diestros y sólo en los rangos de 40 a 44 y de 45 a 49 los zurdos superaron en fuerza a los diestros (gráfica 4).

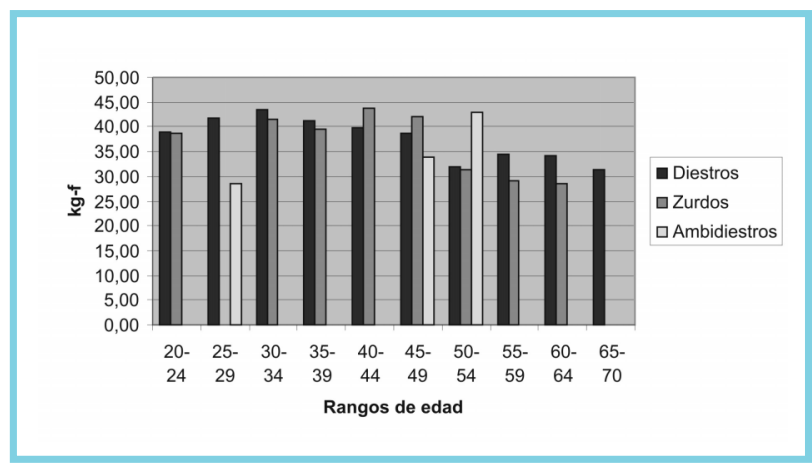

Gráfica 4. Fuerza en posición A de la mano izquierda en hombres por dominancia

En lo que hace referencia a fuerza de agarre y ocupación, al contrario de lo que dicen los estudios previos, en este trabajo no se encontró una clara relación entre la fuerza de agarre y la ocupación que implique trabajo manual ligero, pesado o no manual (gráficas 5 y 6).

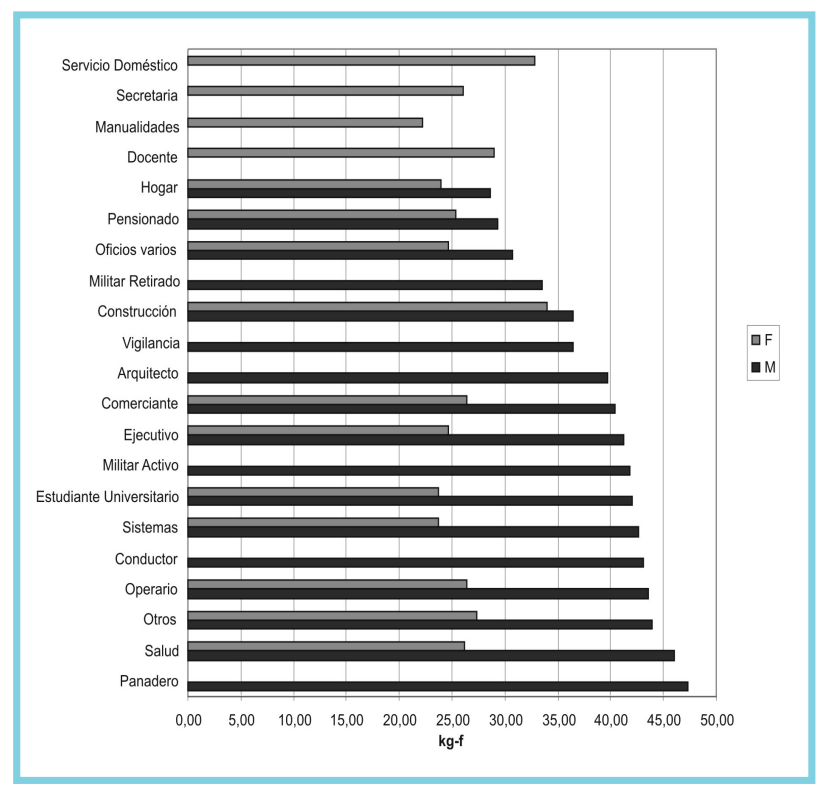

Gráfica 5. Fuerza en la posición A en mano derecha según ocupación y género

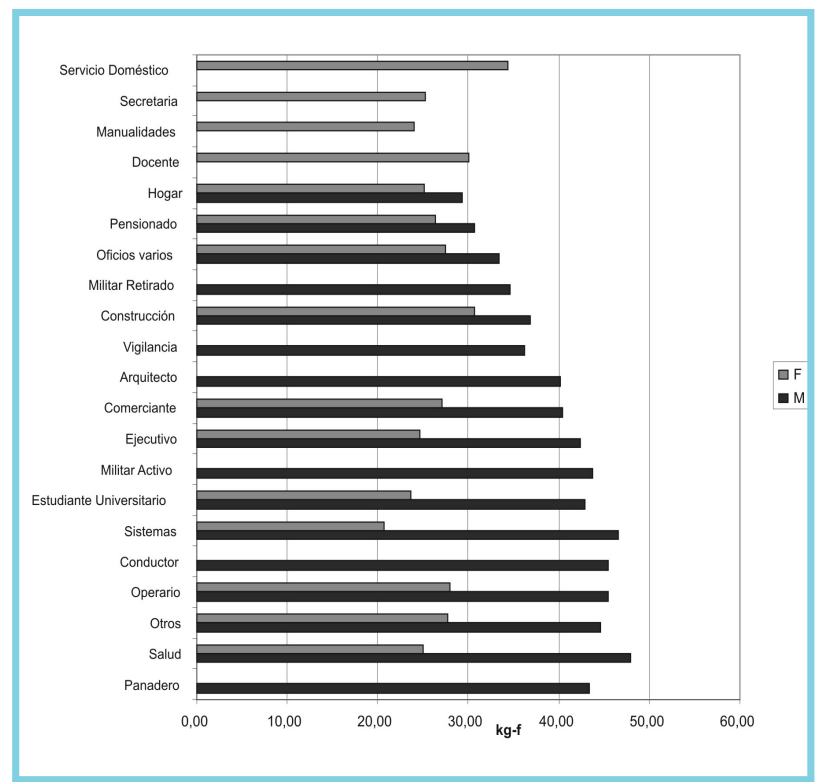

Gráfica 6. Fuerza en la posición B en mano derecha según ocupación y género

Con relación al peso, se encontró que a mayor peso mayor fuerza de agarre en ambos géneros y en ambas posiciones A y B (gráficas 7 y 8).

Con relación a la talla, también, a mayor talla mayor fuerza del agarre en ambos géneros y en ambas posiciones (gráficas 9 y 10): 


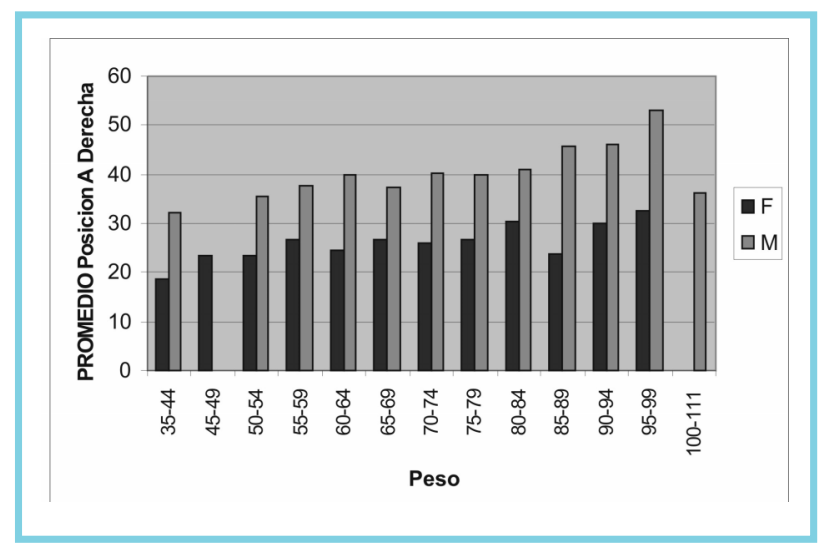

Gráfica 7. Fuerza de agarre en posición A mano derecha según peso y género

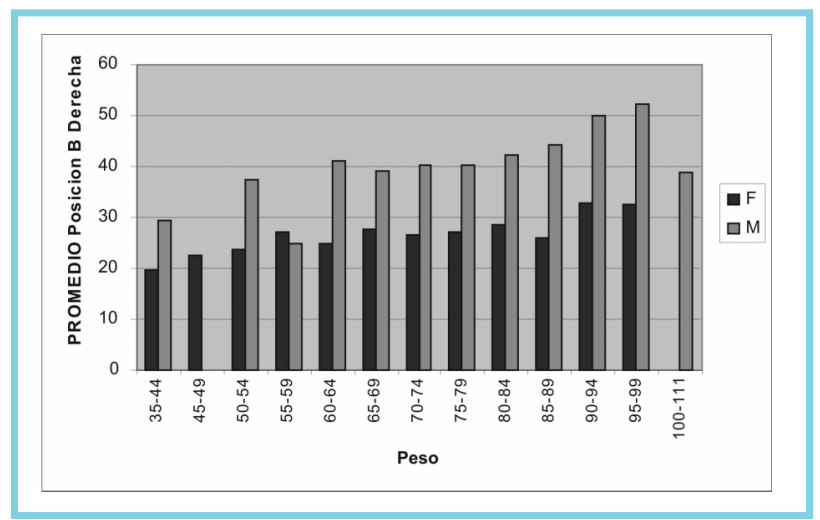

Gráfica 8. Fuerza de agarre en posición B mano derecha según peso y género

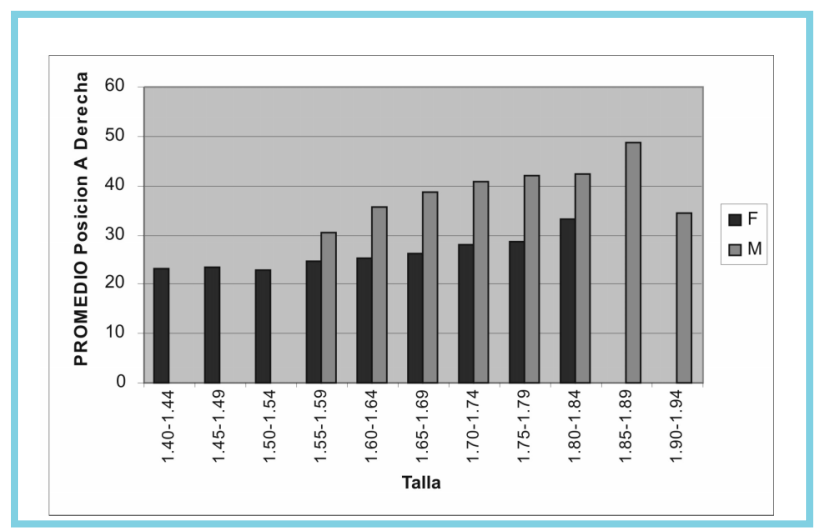

Gráfica 9. Fuerza de agarre en posición A mano derecha según talla y género

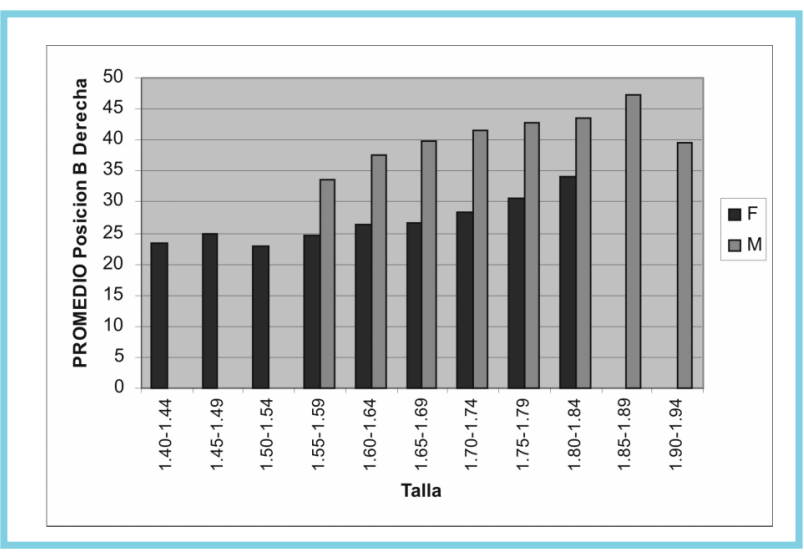

Gráfica 10. Fuerza de agarre en posición B mano derecha según talla y género

Como resultado final se presentan dos tablas (tablas 2 y 3) en las que se pueden apreciar los estándares de la fuerza de agarre en las posiciones A con el codo flexionado y B con el codo extendido.

Al comparar la fuerza de agarre comparativamente entre la posición A con el codo flexionado que es la recomendada por la Asociación Americana de Cirugía de la Mano y la posición B con el codo extendido, se observan valores ligeramente más altos en la posición $\mathrm{B}$.

Se puede observar un descenso en la fuerza de agarre con el aumento de la edad, lo cual corresponde a los estudios previos que se han hecho.

En las tablas también se puede apreciar que la mano derecha tiene ligeramente mayor fuerza que la izquierda.

\section{Discusión y conclusiones}

Como conclusión se presenta una tabla comparativa entre los promedios de la fuerza de agarre de la muestra de población norteamericana y colombiana. Se tomó como base la tabla de Mathiowetz, realizando la conversión de libras a kilogramos.

En la tabla 4 se puede observar que la muestra de población colombiana presenta menor fuerza de 
TABLA 2

ESTÁNDARES DE FUERZA DE AGARRE EN KILOGRAMOS EN LA POSICIÓN A

\begin{tabular}{|c|c|c|c|c|c|c|c|c|c|c|c|}
\hline \multirow[b]{2}{*}{ Edad } & \multirow[b]{2}{*}{ Mano } & \multicolumn{5}{|l|}{ Mujeres } & \multicolumn{5}{|l|}{ Hombres } \\
\hline & & $\begin{array}{l}\text { Promedio } \\
\text { posición A }\end{array}$ & $\begin{array}{l}\text { Desviación } \\
\text { estándar }\end{array}$ & $\begin{array}{l}\text { Error } \\
\text { estándar }\end{array}$ & $\begin{array}{l}\text { Fuerza } \\
\text { minima }\end{array}$ & $\begin{array}{l}\text { Fuerza } \\
\text { maxima }\end{array}$ & $\begin{array}{l}\text { Promedio } \\
\text { posición A }\end{array}$ & $\begin{array}{l}\text { Desviación } \\
\text { estándar }\end{array}$ & $\begin{array}{l}\text { Error } \\
\text { estándar }\end{array}$ & $\begin{array}{l}\text { Fuerza } \\
\text { minima }\end{array}$ & $\begin{array}{l}\text { Fuerza } \\
\text { máxima }\end{array}$ \\
\hline $20-24$ & $\begin{array}{l}\mathrm{D} \\
\mathrm{I}\end{array}$ & $\begin{array}{l}24,05 \\
22,1\end{array}$ & $\begin{array}{l}5,91 \\
5,65\end{array}$ & $\begin{array}{l}1,03 \\
0,98\end{array}$ & $\begin{array}{l}14 \\
12\end{array}$ & $\begin{array}{l}38 \\
34\end{array}$ & $\begin{array}{l}41,48 \\
39,06\end{array}$ & $\begin{array}{l}9,12 \\
7,9\end{array}$ & $\begin{array}{l}2,04 \\
1,76\end{array}$ & $\begin{array}{l}30 \\
27\end{array}$ & $\begin{array}{l}61 \\
52\end{array}$ \\
\hline $25-29$ & $\begin{array}{l}\text { D } \\
\text { I }\end{array}$ & $\begin{array}{l}26,2 \\
23,95\end{array}$ & $\begin{array}{l}4,75 \\
5,03\end{array}$ & $\begin{array}{l}1,18 \\
1,25\end{array}$ & $\begin{array}{l}18 \\
14\end{array}$ & $\begin{array}{l}38 \\
33\end{array}$ & $\begin{array}{l}44,87 \\
41,07\end{array}$ & $\begin{array}{l}8,11 \\
8,2\end{array}$ & $\begin{array}{l}1,91 \\
1,93\end{array}$ & $\begin{array}{l}31 \\
28\end{array}$ & $\begin{array}{l}58 \\
57\end{array}$ \\
\hline $30-34$ & $\begin{array}{l}\mathrm{D} \\
\mathrm{I}\end{array}$ & $\begin{array}{l}27,66 \\
25,82\end{array}$ & $\begin{array}{l}5,68 \\
5,02\end{array}$ & $\begin{array}{l}1,37 \\
1,21\end{array}$ & $\begin{array}{l}18 \\
17\end{array}$ & $\begin{array}{l}40 \\
37\end{array}$ & $\begin{array}{l}44,83 \\
43,4\end{array}$ & $\begin{array}{l}7,52 \\
6,78\end{array}$ & $\begin{array}{l}1,77 \\
1,59\end{array}$ & $\begin{array}{l}28 \\
31\end{array}$ & $\begin{array}{l}54 \\
52\end{array}$ \\
\hline $35-39$ & $\begin{array}{l}\mathrm{D} \\
\mathrm{I}\end{array}$ & $\begin{array}{l}27,68 \\
26,28\end{array}$ & $\begin{array}{l}3,98 \\
4,8\end{array}$ & $\begin{array}{l}0,83 \\
1\end{array}$ & $\begin{array}{l}22 \\
18\end{array}$ & $\begin{array}{l}38 \\
34\end{array}$ & $\begin{array}{l}44,2 \\
41,09\end{array}$ & $\begin{array}{l}8,74 \\
8,58\end{array}$ & $\begin{array}{l}1,9 \\
1,87\end{array}$ & $\begin{array}{l}26 \\
23\end{array}$ & $\begin{array}{l}61 \\
54\end{array}$ \\
\hline $40-44$ & $\begin{array}{l}\text { D } \\
\text { I }\end{array}$ & $\begin{array}{l}27,41 \\
24,74\end{array}$ & $\begin{array}{l}5,09 \\
5,12\end{array}$ & $\begin{array}{l}1,23 \\
1,24\end{array}$ & $\begin{array}{l}20 \\
16 \\
\end{array}$ & $\begin{array}{l}39 \\
36 \\
\end{array}$ & $\begin{array}{l}43,3 \\
40,43 \\
\end{array}$ & $\begin{array}{l}9,19 \\
7,24\end{array}$ & $\begin{array}{l}1,87 \\
1,47\end{array}$ & $\begin{array}{l}21 \\
24 \\
\end{array}$ & $\begin{array}{l}58 \\
52 \\
\end{array}$ \\
\hline $45-49$ & $\begin{array}{l}\mathrm{D} \\
\mathrm{I}\end{array}$ & $\begin{array}{l}25,07 \\
24,33\end{array}$ & $\begin{array}{l}3,75 \\
3,78 \\
\end{array}$ & $\begin{array}{l}0,82 \\
0,82\end{array}$ & $\begin{array}{l}18 \\
18\end{array}$ & $\begin{array}{l}30 \\
31 \\
\end{array}$ & $\begin{array}{l}40,9 \\
38,54 \\
\end{array}$ & $\begin{array}{l}9,21 \\
6,54 \\
\end{array}$ & $\begin{array}{l}2,77 \\
1,97\end{array}$ & $\begin{array}{l}21 \\
27\end{array}$ & $\begin{array}{l}54 \\
49 \\
\end{array}$ \\
\hline $50-54$ & $\begin{array}{l}\mathrm{D} \\
\mathrm{I}\end{array}$ & $\begin{array}{l}23,66 \\
23,33\end{array}$ & $\begin{array}{l}4,09 \\
4,74\end{array}$ & $\begin{array}{l}0,87 \\
1,01\end{array}$ & $\begin{array}{l}17 \\
16\end{array}$ & $\begin{array}{l}32 \\
32\end{array}$ & $\begin{array}{l}35,78 \\
32,85\end{array}$ & $\begin{array}{l}6,32 \\
6,52\end{array}$ & $\begin{array}{l}1,31 \\
1,35\end{array}$ & $\begin{array}{l}28 \\
19\end{array}$ & $\begin{array}{l}48 \\
44\end{array}$ \\
\hline 55-59 & $\begin{array}{l}\mathrm{D} \\
\mathrm{I}\end{array}$ & $\begin{array}{l}23,84 \\
22,6\end{array}$ & $\begin{array}{l}2,99 \\
3,18\end{array}$ & $\begin{array}{l}0,9 \\
0,96\end{array}$ & $\begin{array}{l}19 \\
17 \\
\end{array}$ & $\begin{array}{l}29 \\
28\end{array}$ & $\begin{array}{l}35,33 \\
33,41\end{array}$ & $\begin{array}{l}7,61 \\
8,07\end{array}$ & $\begin{array}{l}1,9 \\
2,01\end{array}$ & $\begin{array}{l}26 \\
18 \\
\end{array}$ & $\begin{array}{l}51 \\
49\end{array}$ \\
\hline $60-64$ & $\begin{array}{l}\mathrm{D} \\
\mathrm{I}\end{array}$ & $\begin{array}{l}24,4 \\
23,55\end{array}$ & $\begin{array}{l}3,71 \\
2,62\end{array}$ & $\begin{array}{l}0,95 \\
0,67\end{array}$ & $\begin{array}{l}19 \\
20\end{array}$ & $\begin{array}{l}32 \\
30\end{array}$ & $\begin{array}{l}34,58 \\
33,83\end{array}$ & $\begin{array}{l}7,25 \\
7,2\end{array}$ & $\begin{array}{l}1,81 \\
1,8\end{array}$ & $\begin{array}{l}21 \\
21\end{array}$ & $\begin{array}{l}45 \\
47\end{array}$ \\
\hline $65-70$ & $\begin{array}{l}\text { D } \\
\text { I }\end{array}$ & $\begin{array}{l}22,05 \\
21,09\end{array}$ & $\begin{array}{l}4,8 \\
5,11\end{array}$ & $\begin{array}{l}0,98 \\
1,04\end{array}$ & $\begin{array}{l}13 \\
10\end{array}$ & $\begin{array}{l}30 \\
32\end{array}$ & $\begin{array}{l}33,07 \\
31,47\end{array}$ & $\begin{array}{l}8,43 \\
8,14\end{array}$ & $\begin{array}{l}1,93 \\
1,86\end{array}$ & $\begin{array}{l}22 \\
21\end{array}$ & $\begin{array}{l}53 \\
48\end{array}$ \\
\hline
\end{tabular}

agarre comparativamente. Esta comparación se realizó únicamente en la posición A, pues sólo se cuenta con los estudios de Mathiowetz en esta posición.

En la gráfica 11 se puede apreciar claramente la curva descendente en la fuerza del agarre con el aumento de la edad en los hombres tanto norteamericanos como colombianos.

Se encontró también que el comportamiento de la fuerza del agarre en la muestra femenina colombiana es mucho más estable comparada con la muestra femenina norteamericana.

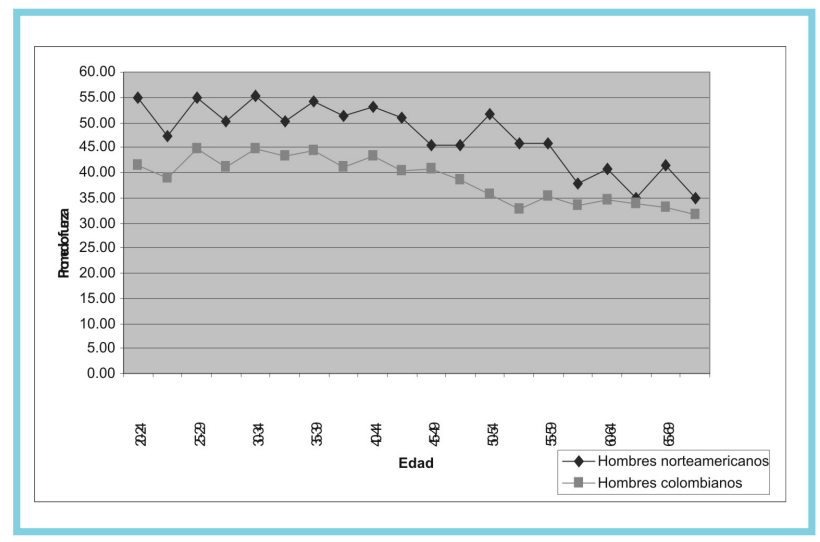

Grafica 11. Comparación de fuerza de agarre en posición A de hombres Norteamericanos y Colombianos 
TABla 3

ESTÁNDARES DE FUERZA DE AGARRE EN KILOGRAMOS EN LA POSICIÓN B

\begin{tabular}{|c|c|c|c|c|c|c|c|c|c|c|c|}
\hline \multirow[b]{2}{*}{ Edad } & \multirow[b]{2}{*}{ Mano } & \multicolumn{5}{|l|}{ Mujeres } & \multicolumn{5}{|l|}{ Hombres } \\
\hline & & $\begin{array}{l}\text { Promedio } \\
\text { posición A }\end{array}$ & $\begin{array}{l}\text { Desviación } \\
\text { estándar }\end{array}$ & $\begin{array}{l}\text { Error } \\
\text { estándar }\end{array}$ & $\begin{array}{l}\text { Fuerza } \\
\text { minima }\end{array}$ & $\begin{array}{l}\text { Fuerza } \\
\text { maxima }\end{array}$ & $\begin{array}{l}\text { Promedio } \\
\text { posición A }\end{array}$ & $\begin{array}{l}\text { Desviación } \\
\text { estándar }\end{array}$ & $\begin{array}{l}\text { Error } \\
\text { estándar }\end{array}$ & $\begin{array}{l}\text { Fuerza } \\
\text { minima }\end{array}$ & $\begin{array}{l}\text { Fuerza } \\
\text { máxima }\end{array}$ \\
\hline \multirow[t]{2}{*}{$20-24$} & D & 24,21 & 5,16 & 0,89 & 13 & 36 & 42,28 & 8,81 & 1,97 & 29 & 58 \\
\hline & I & 22,56 & 5,16 & 0,89 & 14 & 34 & 40,13 & 6,83 & 1,52 & 28 & 51 \\
\hline \multirow[t]{2}{*}{$25-29$} & D & 26,75 & 5,47 & 1,36 & 18 & 40 & 45,16 & 7,49 & 1,76 & 33 & 56 \\
\hline & I & 24 & 5,42 & 1,35 & 16 & 37 & 41,29 & 7,06 & 1,66 & 29 & 53 \\
\hline \multirow{2}{*}{$30-34$} & D & 27,82 & 6.03 & 1,46 & 17 & 42 & 4651 & 8.41 & 198 & 30 & 60 \\
\hline & I & 26,13 & 5,17 & 1,25 & 18 & 35 & 45,24 & 7,56 & 1,78 & 29 & 60 \\
\hline \multirow[t]{2}{*}{ 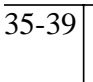 } & $\mathrm{D}$ & 28,42 & 5,98 & 1,24 & 20 & 40 & 46,12 & 7,95 & 1,73 & 29 & 59 \\
\hline & I & 26,71 & 6,09 & 1,27 & 16 & 37 & 42,55 & 7,83 & 1,7 & 28 & 52 \\
\hline \multirow{3}{*}{$\overline{40-44}$} & & & & & & & & & & & \\
\hline & D & 27,64 & $\begin{array}{l}4,73 \\
478\end{array}$ & 1,14 & 16 & 34 & $\begin{array}{l}45,18 \\
4155\end{array}$ & 8,18 & 1,67 & 27 & $\begin{array}{l}58 \\
53\end{array}$ \\
\hline & 1 & 25,6 & 4,78 & 1,16 & 15 & 34 & 41,55 & 6,36 & 1,29 & 29 & 53 \\
\hline \multirow[t]{2}{*}{$45-49$} & $\mathrm{D}$ & 25,55 & 4,48 & 0,97 & 18 & 33 & 42,12 & 11,43 & 3,44 & 20 & 56 \\
\hline & I & 25,17 & 3,94 & 0,85 & 18 & 31 & 39,06 & 5,17 & 1,55 & 26 & 47 \\
\hline \multirow[t]{2}{*}{$50-54$} & D & 24,86 & 4,73 & 1 & 17 & 32 & 36,1 & 8,68 & 1,81 & 21 & 52 \\
\hline & $\mathrm{I}$ & 23,69 & 5,18 & 1,1 & 15 & 32 & 33,52 & 7,05 & 1,47 & 21 & 49 \\
\hline \multirow[t]{2}{*}{ 55-59 } & D & 25,96 & 3,18 & 0,96 & 22 & 33 & 35,02 & 7,19 & 1,79 & 26 & 48 \\
\hline & I & 22,69 & 3,33 & 1 & 18 & 30 & 33,41 & 8,28 & 2,07 & 16 & 49 \\
\hline \multirow[t]{2}{*}{$60-64$} & D & 24,51 & 3,8 & 0,98 & 18 & 32 & 36,45 & 7,18 & 1.79 & 23 & 49 \\
\hline & I & 24,08 & 3,77 & 0,97 & 19 & 34 & 34,43 & 7,81 & 1,95 & 24 & 49 \\
\hline \multirow[t]{2}{*}{$65-70$} & D & 22,31 & 5,05 & 1,03 & 12 & 35 & 33,94 & 8,03 & 1,84 & 25 & 53 \\
\hline & I & 21,47 & 4,58 & 0,93 & 14 & 31 & 32,57 & 7,88 & 1,8 & 23 & 50 \\
\hline
\end{tabular}

Considerando que hasta la fecha en las evaluaciones de la fuerza de agarre se tomaban como parámetros las mediciones de población norteamericana, resulta valioso contar con estándares propios de nuestra población, con fines, tanto de diagnóstico como de pronóstico.
Se recomienda establecer estándares en otras muestras de población, otras localidades, ampliar la muestra y ampliar el rango de edad. De la misma manera investigar más profundamente la relación entre ocupación y fuerza de agarre que en el presente estudio no resulta consistente. 


\section{TABLA 5}

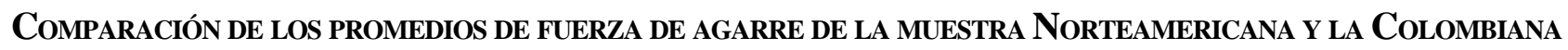

\begin{tabular}{|c|c|c|c|c|c|}
\hline Edad & Mano & $\begin{array}{c}\text { Hombres } \\
\text { norteamericanos }\end{array}$ & $\begin{array}{c}\text { Hombres } \\
\text { colombianos }\end{array}$ & $\begin{array}{c}\text { Mujeres } \\
\text { norteamericanas }\end{array}$ & $\begin{array}{c}\text { Mujeres } \\
\text { colombianas }\end{array}$ \\
\hline $20-24$ & $\begin{array}{l}\mathrm{D} \\
\mathrm{I}\end{array}$ & $\begin{array}{l}50,89 \\
47,40 \\
\end{array}$ & $\begin{array}{l}41,48 \\
39,06 \\
\end{array}$ & $\begin{array}{l}31,93 \\
27,67 \\
\end{array}$ & $\begin{array}{l}24,05 \\
22,1\end{array}$ \\
\hline $25-29$ & $\begin{array}{l}\mathrm{D} \\
\mathrm{I}\end{array}$ & $\begin{array}{l}54,79 \\
50,12 \\
\end{array}$ & $\begin{array}{l}44,87 \\
41,07 \\
\end{array}$ & $\begin{array}{l}33,79 \\
28,80 \\
\end{array}$ & $\begin{array}{l}26,2 \\
23,95 \\
\end{array}$ \\
\hline $30-34$ & $\begin{array}{l}\text { D } \\
\text { I }\end{array}$ & $\begin{array}{l}55,16 \\
50,08\end{array}$ & $\begin{array}{l}44,83 \\
43,4\end{array}$ & $\begin{array}{l}34,79 \\
30,84\end{array}$ & $\begin{array}{l}27,66 \\
25,82\end{array}$ \\
\hline $35-39$ & $\begin{array}{l}\mathrm{D} \\
\mathrm{I}\end{array}$ & $\begin{array}{l}54,30 \\
51,21 \\
\end{array}$ & $\begin{array}{l}44,2 \\
41,09 \\
\end{array}$ & $\begin{array}{l}33,61 \\
30,07 \\
\end{array}$ & $\begin{array}{l}27,68 \\
26,28 \\
\end{array}$ \\
\hline $40-44$ & $\begin{array}{l}\mathrm{D} \\
\mathrm{I}\end{array}$ & $\begin{array}{l}52,98 \\
51,08 \\
\end{array}$ & $\begin{array}{l}43,3 \\
40,43 \\
\end{array}$ & $\begin{array}{l}31,93 \\
28,26 \\
\end{array}$ & $\begin{array}{l}27,41 \\
24,74 \\
\end{array}$ \\
\hline $45-49$ & $\begin{array}{l}\mathrm{D} \\
\mathrm{I}\end{array}$ & $\begin{array}{l}45,36 \\
45,36 \\
\end{array}$ & $\begin{array}{l}40,9 \\
38,54 \\
\end{array}$ & $\begin{array}{l}28,21 \\
25,40 \\
\end{array}$ & $\begin{array}{l}25,07 \\
24,33 \\
\end{array}$ \\
\hline $50-54$ & $\begin{array}{l}\mathrm{D} \\
\mathrm{I}\end{array}$ & $\begin{array}{l}51,53 \\
45,81 \\
\end{array}$ & $\begin{array}{l}35,78 \\
32,85 \\
\end{array}$ & $\begin{array}{l}29,85 \\
25.99 \\
\end{array}$ & $\begin{array}{l}23,66 \\
23,33 \\
\end{array}$ \\
\hline $55-59$ & $\begin{array}{l}\mathrm{D} \\
\mathrm{I}\end{array}$ & $\begin{array}{l}45,86 \\
37,74 \\
\end{array}$ & $\begin{array}{l}35,33 \\
33,41 \\
\end{array}$ & $\begin{array}{l}25,99 \\
21,46 \\
\end{array}$ & $\begin{array}{l}23,84 \\
22,6\end{array}$ \\
\hline $60-64$ & $\begin{array}{l}\text { D } \\
\text { I }\end{array}$ & $\begin{array}{l}40,69 \\
34,84 \\
\end{array}$ & $\begin{array}{r}34,58 \\
33,83 \\
\end{array}$ & $\begin{array}{l}24,99 \\
20,73\end{array}$ & $\begin{array}{l}24,4 \\
23,55\end{array}$ \\
\hline $65-69$ & $\begin{array}{l}\mathrm{D} \\
\mathrm{I}\end{array}$ & $\begin{array}{l}41,39 \\
34,84\end{array}$ & $\begin{array}{l}33,07 \\
31,47\end{array}$ & $\begin{array}{l}22,50 \\
18,60\end{array}$ & $\begin{array}{c}22,05 \\
2109\end{array}$ \\
\hline
\end{tabular}

Recibido: Abril 2007

Aceptado: Junio 2007

\section{Referencias}

American Society for Surgery of the Hand The hand. Examination and diagnosis. Estados Unidos. Churchill Livingstone. 1990.

Balogun, J. Grip strength: effects of testing posture and elbow position. Archives of Physical Medicine and Rehabilitation. April, 1991; 72, 280-283.

Blanco, J.; Maya, J. Fundamentos de salud pública. "Epidemiología básica y principios de investigación”. Medellín: Corporación para Investigaciones Biológicas, (CIB). 1999.
Chwen, Y.S. Grip strength in differents positions of elbow and shoulder. Archives of Physical Medicine and Rehabilitation, Jul. 1994; 75.

Conti, G. Clinical interpretation of "Grip strengths and required forces in accessing everyday containers in a normal population". The American Journal of Occupational Therapy, Sept., 1998; 52 (8): 627-628.

Daniel, W. Bioestadística. Base para el análisis de las ciencias de la salud. México. Limusa. 1989.

Desrosiers, J., Bravo, G. Normative data for grip strength of elderly men and women. The American Journal of Occupational Therapy, Jul.1995; 49 (7): 637-642.

Fiebert, I. Dynamometric grip strength assessment of subjects sixty years and older. Physical 
and Occupational Therapy in Geriatrics, 1995; 13 (4): 27-39.

Giraldo, J. Fuerza de agarre en hombres ancianos ambulatorios. Revista Asociación Colombiana de Gerontología y Geriatría, 2003; 17 (1): 455-460.

Härkönen, R. Grip strength and hand position of the dynamometer in 204 finish adults. The Journal of Hand Surgery, Feb., 1993; 18 (1): 129-132.

Heyward, V. Evaluación y prescripción del ejercicio. Editorial Paidotribo, España. 2001.

Hunter, J.M. et al. Rehabilitation of the hand: Surgery and therapy. CV Mosby, St Louis, 4a. ed. 1995.

Josty, I.C. Grip and pinch strength variations in different types of workers. Journal of Hand Surgery. British and European Volume, 1997; 22B, 266-268.

Kapandji, A.I. Fisiología articular. España. Editorial Médica Panamericana - Maloine. 1998

Kellor, M.; Frostt J.; Silverberg, N. Hand strength on dexterity. Norms for Clinical use. American Journal of Occupational Therapy. 1971.

Kuzala, E., Vargo, M. The relationship between elbow position and grip strength. The American Journal of Occupational Therapy, Jun., 1992; 46 (6): 509-512.

Mathiowetz, V. Grip and pinch strength: Normative data for adults. Archives of physical medicine and rehabilitation, 1985; 66, 69-72.

Ministerio de Salud. República de Colombia. Normas científicas, técnicas y administrativas para la investigación en salud. Resolución No 008430 de 1993. Bogotá, 1993.

Nitschke, J. When is a change a genuine change? A clinically meaningful interpretation of grip strength measurements in healthy and disabled women. Journal of Hand Therapy. Enero-Marzo, 1999; 25-29.

Nurgul, A. Grip strength: Effect of hand dominance. The Singapore Med Journal. 2002; 43 (5): 237.

Peddeti, L. Occupational therapy the C.V. Mosby Company, 3a. ed., 1990.

Petersen, P., Petrick, M., Connor, H. Grip strength and hand dominance: Challenging the $10 \%$ rule. The American Journal of Occupational Therapy, Jul., 1989; 43 (7): 444-447.

Polit, D., Hungler, B. Investigación científica en ciencias de la salud. México: McGraw-Hill. 2000.

Rantanen, T. Midlife hand grip strength as a predictor of old age disability. Journal American Medicine Association, Feb., 1999; 281 (6): 558-560.

Rheallth, W. Interster reliability of the hand-held dynamometer for wrist flexion and extension. Archives of physical medicine and rehabilitation, Dic., 1989; 70, 907-909.

Rice, M., Leonard, C., Carter, M. Grip strengths and required forces in accessing everyday containers in a normal population. The American Journal of Occupational Therapy, Sept., 1998; 52 (8): 621-622.

Sleder, R. The hand examination an diagnosis. American Society for surgery of the Hand. 3a. ed. 1990.

Stanley, B. Concepts in hand rehabilitation. F.A. Davis Company. Philadelphia. 1992.

Tamayo, S y Tamayo, M. El proceso de la investigación científica. Fundamentos de investigación con manual de evaluación de proyectos. México: Noriega Editores. 1993. 
